\title{
Video Article \\ An Efficient Method for the Synthesis of Peptoids with Mixed Lysine-type/ Arginine-type Monomers and Evaluation of Their Anti-leishmanial Activity
}

\author{
Hannah L. Bolt ${ }^{1}$, Paul W. Denny ${ }^{2}$, Steven L. Cobb ${ }^{1}$ \\ ${ }^{1}$ Department of Chemistry, Durham University \\ ${ }^{2}$ School of Medicine, Pharmacy and Health, Durham University
}

Correspondence to: Hannah L. Bolt at h.I.bolt@durham.ac.uk, Steven L. Cobb at s.I.cobb@durham.ac.uk

URL: https://www.jove.com/video/54750

DOI: doi: $10.3791 / 54750$

Keywords: Biochemistry, Issue 117, peptoid, synthesis, arginine monomer, lysine monomer, amphipathic, parasite, Leishmaniasis

Date Published: 11/2/2016

Citation: Bolt, H.L., Denny, P.W., Cobb, S.L. An Efficient Method for the Synthesis of Peptoids with Mixed Lysine-type/Arginine-type Monomers and Evaluation of Their Anti-leishmanial Activity. J. Vis. Exp. (117), e54750, doi:10.3791/54750 (2016).

\section{Abstract}

This protocol describes the manual solid-phase synthesis of linear peptoids that contain two differently functionalized cationic monomers. In this procedure amino functionalized 'lysine' and guanido functionalized 'arginine' peptoid monomers can be included within the same peptoid sequence. This procedure uses on-resin ( $N$-(1-(4,4-dimethyl-2,6-dioxocyclohexylidene)ethyl) or Dde protection, orthogonal conditions to the Boc protection of lysine monomers. Subsequent deprotection allows an efficient on-resin guanidinylation reaction to form the arginine residues. The procedure is compatible with the commonly used submonomer method of peptoid synthesis, allowing simple peptoids to be made using common laboratory equipment and commercially available reagents. The representative synthesis, purification and characterization of two mixed peptoids is described. The evaluation of these compounds as potential anti-infectives in screening assays against Leishmania mexicana is also described. The protozoan parasite L. mexicana is a causative agent of cutaneous leishmaniasis, a neglected tropical disease that affects up to 12 million people worldwide.

\section{Video Link}

The video component of this article can be found at https://www.jove.com/video/54750/

\section{Introduction}

Peptoids (or poly- $N$-substituted glycines) are a class of peptide-mimetics that offer similar properties to peptides and as such are increasingly being investigated for medicinal and materials applications. In peptides, the side chain of each amino acid is connected to the $\alpha$-carbon of the amide backbone; in peptoids the side chains are shifted onto the nitrogen atom of the backbone. Crucially, this gives peptoids greater resistance to proteolysis.

Peptoids are commonly synthesized using the submonomer method pioneered by Zuckermann et al., where peptoid monomers can be built by sequential haloacetylation of an amine functionality attached to a solid support and subsequent displacement of the halogen using a primary amine. ${ }^{1}$ Our group has recently developed an adaptation to this submonomer method to allow lysine- and arginine-type peptoid residues to be included within the same peptoid sequence for the first time. ${ }^{2}$ This manual solid-phase approach to peptoid synthesis uses commercially available reagents and common laboratory equipment, making it accessible for the majority of laboratories. Peptoids have been shown to have promising activities against a wide range of Gram negative bacteria, Gram positive bacterial and fungal species that are comparable to many known antimicrobial peptides. ${ }^{3-9}$

In our work, peptoids have been used as novel anti-infective compounds for treatment of the neglected tropical disease leishmaniasis. ${ }^{5,10}$ Leishmaniasis is endemic in more than 80 countries worldwide and it is estimated that over 12 million people are infected globally. ${ }^{11}$ The disease is caused by protozoan parasites that are transmitted by the bite of a sandfly. Leishmania species can cause cutaneous leishmaniasis, a condition that leads to scarring and damage to mucous membranes, or the life-threatening visceral leishmaniasis, which causes fatal organ damage. No vaccine is currently available for this disease and existing treatments rely on a small number of drugs that have severe side effects. In addition, resistance to existing drugs is an emerging and serious problem so new treatments are desperately needed to effectively treat leishmaniasis in the future. ${ }^{12-16}$

In these antimicrobial applications, peptoids are often designed to be amphipathic with a mixture of cationic and hydrophobic monomers. ${ }^{3,4}$ This can give peptoids a degree of selectivity towards bacterial cells, reduce toxicity to mammalian cells, and to improve their activity as molecular transporters. ${ }^{17-20}$ The majority of the anti-infective peptoids in the literature contain cationic side chains that are exclusively comprised of either amino functionalized lysine-type monomers or arginine-type residues. Peptide-peptoid chimeras, where the cationic chains are comprised of the amino acids lysine or arginine, have also been synthesized to examine the effect of cationic groups on activity and toxicity. ${ }^{21-25}$

Poly-lysine peptoids can be easily synthesized using commercially available Boc-protected amines. The poly-arginine peptoids reported can be made using a method which uses pyrazole-1-carboxamidine as a guanidinylation agent. ${ }^{18}$ However, this can only be undertaken after the peptoid 
has been cleaved from the resin and Boc protection on side chains removed, so every lysine-type residue within the sequence is transformed into an arginine residue. In an effort to fine tune the chemical and biological properties of the compounds, we developed a method that allows dual cationic functionality (e.g., NLys and NArg) to be included in any given peptoid sequence for the first time. ${ }^{2}$

Herein, we describe the synthesis, purification and characterization of two novel peptoids that contain both lysine- and arginine-type residues in the same sequence. The method uses orthogonal $\mathrm{N}$-Boc and $\mathrm{N}$-Dde protection on resin with pyrazole-1-carboxamidine as a guanidinylation reagent. The biological evaluation of these peptoids is also described in cytotoxicity assays against Leishmania mexicana, the causative agent of cutaneous leishmaniasis. This provides a practical method to access peptoids with dual cationic functionality and to assess their biological activity. It is expected that this method will aid the synthesis of amphipathic peptoids by the peptoid community in the future.

\section{Protocol}

\section{Solid-phase Synthesis of Peptoids}

NOTE: Peptoids are synthesized manually using the submonomer procedure of solid-phase peptoid synthesis. This method allows high coupling efficiency and good final product yields. Synthesis on solid-phase also allows excess reactants to be removed easily at the end of each step and the method has been modified here to allow different functionalized cationic monomers (i.e., arginine-type and lysine-type residues) to be included within the same sequence. ${ }^{1,2}$

\section{Synthesis of a linear peptoid}

Caution: Carry out safety assessments before starting synthesis. Carry out all reactions in a fume hood and wear adequate personal protective equipment as appropriate (i.e., disposable nitrile gloves, safety glasses and a lab coat). Take particular care when using the following reagents and solvents. Dimethylformamide (DMF) is a suspected teratogen and dichloromethane (DCM) is a carcinogen. $N, N$ 'diisopropylcarbodiimide (DIC) and piperidine are hazardous to eyes, skin, via respiratory inhalation and may cause skin sensitization. Hydrazine is a suspected carcinogen, fatal if inhaled and causes severe burns to skin or eyes. Bromoacetic acid is also hazardous to skin, eyes and the respiratory tract and may cause burns upon contact. Trifluoroacetic acid (TFA) is a volatile liquid and can cause severe burns so handle with care. Heavy duty gloves are recommended.

1. Add $0.12 \mathrm{~g}$ Fmoc-protected Rink Amide resin $(0.1 \mathrm{mmol}$, typical loading $0.7 \mathrm{mmol} / \mathrm{g})$ to a capped $20 \mathrm{ml}$ polypropylene reaction vessel with two frits. Add $5 \mathrm{ml}$ dimethylformamide (DMF) to swell the resin and leave the vessel to stand for at least 60 minutes at room temperature. Drain the DMF using a solid-phase extraction vacuum platform.

2. To deprotect the Fmoc group on the swollen resin, add $2 \mathrm{ml}$ piperidine solution ( $20 \%$ in DMF v/v). Place the vessel on a shaker platform at room temperature $(450 \mathrm{rpm})$ and shake for $5 \mathrm{~min}$. Remove the solution via vacuum station.

1. Repeat Fmoc deprotection with $2 \mathrm{ml}$ piperidine solution and shake for $15 \mathrm{~min}$ at room temperature. Drain the solution as before.

3. Wash the resin by adding $2 \mathrm{ml} \mathrm{DMF}$ and mixing the resin for $30 \mathrm{sec}$. Drain the DMF and repeat three more times.

4. For the acetylation, add $1 \mathrm{ml}$ of bromoacetic acid solution ( $0.6 \mathrm{M}$ in DMF) and $0.2 \mathrm{ml} N, N^{\prime}$-diisopropylcarbodiimide solution (DIC, $50 \%$ in DMF v/v). Leave reaction vessel to shake for $20 \mathrm{~min}$ at room temperature. Drain the solution and wash the resin with $2 \mathrm{ml}$ DMF three times.

5. For the displacement, add $1 \mathrm{ml}$ of amine solution (1.5 M in DMF). Shake the resin for $60 \mathrm{~min}$ at room temperature. Drain the solution and wash the resin with $2 \mathrm{ml}$ DMF three times.

1. To add an arginine-type monomer, follow step 1.7. Depending upon the desired peptoid sequence, different amines will be added.

6. Repeat steps 1.1.4 and 1.1.5.

7. To include a guanidine functionalized monomer (i.e., NArg), add $1 \mathrm{ml}$ of unprotected diamine solution (1.5 M in DMF) to the resin and shake for $60 \mathrm{~min}$ at room temperature.

1. Drain the solution and wash the resin with $2 \mathrm{ml}$ DMF three times.

2. Add 2-acetyldimedone $(0.2 \mathrm{~g}, 1 \mathrm{mmol}$ in $0.5 \mathrm{ml} \mathrm{DMF}, 10$ equivalents) to add the Dde group to the free primary amine and shake for $60 \mathrm{~min}$ at room temperature. Drain the solution and wash the resin with $2 \mathrm{ml} \mathrm{DMF}$ three times.

8. Continue submonomer synthesis as in 1.1.4 to 1.1.7 until the desired sequence is made. Add $2 \mathrm{ml}$ DMF to wash the resin and repeat three times.

9. To deprotect the Dde group on resin, add $4 \mathrm{ml} 2 \%$ hydrazine solution (in DMF v/v) and shake for 3 min at room temperature. Drain the solution and repeat three times.

10. Drain the solution and wash the resin with $2 \mathrm{ml}$ DMF three times.

11. Add pyrazole-1-carboxamidine (6 equivalents per free amine, i.e., per NArg monomers, in the minimum volume of DMF) and $N, N$ diisopropylethylamine or DIPEA ( 6 equivalents per free amine) and shake at room temperature for $60 \mathrm{~min}$.

12. Drain the solution and wash the resin with $2 \mathrm{ml}$ dichloromethane three times. Leave the resin to dry in air for $10 \mathrm{~min}$ then the resin can be stored until cleavage (section 2).

13. To pause the synthesis, wash the resin with $2 \mathrm{ml}$ DMF three times. Add $2 \mathrm{ml} \mathrm{DMF}$, stopper the synthesis vessel and leave at room temperature in a fume hood.

NOTE: The synthesis may be paused after any displacement step (except the second displacement step as diketopiperazines may be formed).

2. Side-chain deprotection and cleavage from resin

1. Undertake a test cleave to check the progress of synthesis (purity and mass) at any point during synthesis after the displacement steps, addition or removal of Dde-protecting group or after the final sequence has been made.

1. Transfer approximately 10 resin beads from the reaction vessel into a new $8 \mathrm{ml}$ polypropylene fritted cartridge. 
2. Add $1 \mathrm{ml}$ of trifluoroacetic acid cleavage cocktail (containing $95 \% \mathrm{TFA}, 2.5 \% \mathrm{H}_{2} \mathrm{O}, 2.5 \%$ triisopropylsilane) and shake for 90 min at room temperature.

3. Filter the TFA cleavage cocktail from the resin using the fritted reaction vessel into a $10 \mathrm{ml}$ round bottomed flask.

4. Evaporate the cleavage cocktail using a rotary evaporator and redissolve the resulting oil in $1 \mathrm{ml}$ acetonitrile/water for submission to LC-MS or analytical HPLC.

2. For the final cleavage: in the same fritted polypropylene reaction cartridge used for synthesis, add 4 ml of the TFA cleavage cocktail (95\% TFA, 2.5\% $\mathrm{H}_{2} \mathrm{O}, 2.5 \%$ triisopropylsilane) and cover the vessel. Shake for $90 \mathrm{~min}$ at room temperature.

3. Filter the TFA cleavage cocktail from the resin using the fritted reaction vessel into a $50 \mathrm{ml}$ round bottomed flask.

4. Evaporate the cleavage cocktail using a rotary evaporator. After the TFA has been removed, the product should be obtained as an oil. To aid TFA removal from this crude oil, add $2 \mathrm{ml}$ anhydrous diethyl ether and the peptoid should precipitate.

1. Either remove the diethyl ether via pipette and discard or evaporate using a rotary evaporator. Repeat diethyl ether precipitation three times.

5. Dissolve the crude peptoid in $10 \mathrm{ml}$ acetonitrile/acidified water solution ( $50 \%$ acetonitrile, $0.1 \%$ TFA in water v/v). Transfer to a preweighed container, freeze at $20^{\circ} \mathrm{C}$ and lyophilize to a dry powder.

\section{Characterization and Purification}

NOTE: The peptoid synthesis can be monitored and the final peptoid assessed via analytical reverse-phase HPLC using a C18 column and electrospray liquid-chromatography mass spectrometry (LC-MS). All HPLC solvents of solvents for LC-MS should be freshly prepared.

\section{Analytical HPLC}

1. Weigh $1 \mathrm{mg}$ of peptoid in a small glass vial. Add the minimum volume of acetonitrile to dissolve and dilute to $1 \mathrm{ml}$ with water. Ensure that the peptoid has completely dissolved.

2. Inject $10 \mu$ to analytical HPLC (suggested gradient $0-100 \%$ solvent $B$ over 30 min, where solvent $A=95 \%$ water, $5 \%$ acetonitrile, $0.05 \%$ TFA and solvent $B=95 \%$ acetonitrile, $5 \%$ water, $0.03 \%$ TFA), as per manufacturer's instructions.

3. Visualize UV spectrum at $220 \mathrm{~nm}$.

\section{ESI LC-MS}

1. Make $1 \mathrm{mg} / \mathrm{ml}$ peptoid solution as in step 2.1.1.

2. Inject $1 \mu$ to electrospray LC-MS to determine if the molecular weight of the target peptoid is present, using manufacturer's instructions.

3. Check the target mass of the peptoid sequence using a peptoid calculator, as per instructions on the calculator. ${ }^{26}$ This web utility also allows the assignment of any deletion/addition products seen in the mass spectrum. ${ }^{26}$

\section{Preparative reverse phase HPLC}

1. Dissolve crude peptoids into $2 \mathrm{ml}$ acidified water/acetonitrile (95\% water, $5 \% \mathrm{MeCN}, 0.1 \% \mathrm{TFA}$ ) and purify by preparative RP-HPLC using manufacturer's protocol. Determine the gradient by the elution time obtained from analytical HPLC and the amount injected will depend upon the column dimensions.

2. Visualize using a detector set at $220 \mathrm{~nm}$.

3. Collect fractions in $15 \mathrm{ml}$ centrifuge tubes, freeze at $20^{\circ} \mathrm{C}$ and lyophilize.

4. Re-analyze fractions using LC-MS and analytical HPLC according to manufacturer's protocol. Recombine purified fractions.

\section{Biological Testing against Leishmania mexicana Parasites}

Caution: Safety assessments must be carried out before starting synthesis. Leishmania mexicana is classified a hazard group 2 pathogen in the U.K. and adequate control measures must be in place prior to starting testing. All work must be carried out in a class 2 microbiological safety cabinet and adequate personal protective equipment worn as appropriate (i.e., nitrile gloves, safety glasses and a lab coat).

1. Subculture of parasites

1. Defrost $1 \mathrm{ml}-150{ }^{\circ} \mathrm{C}$ frozen stock Leishmania mexicana $\mathrm{M} 379$ by placing vial in a $37{ }^{\circ} \mathrm{C}$ water bath for $30 \mathrm{sec}$.

2. Transfer the stock solution to $10 \mathrm{ml}$ Schneider's Insect medium (at $\mathrm{pH} 7.0$ supplemented with $15 \%$ heat-inactivated fetal bovine serum and $1 \%$ penicillin/streptomycin) in a $25 \mathrm{~cm}^{3}$ cell culture flask with a non-vented cap.

3. Incubate at $26^{\circ} \mathrm{C}$ for $72 \mathrm{hr}$.

4. Examine parasites under microscope (400X magnification) to check condition. They should be insect stage promastigotes, procyclic forms in log phase with many dividing cells.

5. Maintain procyclic promastigotes by sub-culturing parasites to a concentration of $5 \times 10^{5}$ parasites/ml every three days. Count cells using a Neubauer improved hemocytometer.

2. Transformation of L. mexicana insect stage into mammalian stage axenic amastigote form ${ }^{27}$

1. Day 0: Prepare $10 \mathrm{ml}$ culture of log stage parasites at $5 \times 10^{5}$ parasites $/ \mathrm{ml}$ in Schneider's Insect medium (at pH 7.0 supplemented with $15 \%$ heat-inactivated fetal bovine serum and $1 \%$ penicillin/streptomycin) in a $25 \mathrm{~cm}^{3}$ cell culture flask with a non-vented cap.

2. Incubate at $26^{\circ} \mathrm{C}$ for $48 \mathrm{hr}$.

3. Day 3: Transfer $10 \mathrm{ml}$ culture to a $50 \mathrm{ml}$ centrifuge tube and centrifuge at $447 \mathrm{x} \mathrm{g}$ for $5 \mathrm{~min}$.

4. Pour off old medium and add $10 \mathrm{ml}$ Schneider's Insect medium (at pH 5.5 supplemented with $20 \%$ heat-inactivated fetal bovine serum and $1 \%$ penicillin/streptomycin). Gently resuspend the pellet of parasites in the new medium using a pipette.

5. Count the number of parasites using a Neubauer improved hemocytometer. Dilute to a concentration of $5 \times 10^{5} \mathrm{parasites} / \mathrm{ml}$ with pH 5.5 medium and transfer to a $25 \mathrm{~cm}^{3}$ cell culture flask. 
6. Incubate at $26^{\circ} \mathrm{C}$ for approximately 6 days.

7. Day 9: Examine parasites under a microscope (400X). They should be in the non-replicating, infectious metacyclic promastigote stage.

8. Count the number of parasites using a Neubauer improved hemocytometer. Dilute to a concentration of $5 \times 10^{5} \mathrm{parasites} / \mathrm{ml}$ with pH 5.5 medium.

9. Remove $10 \mathrm{ml}$ of cell culture and transfer to cell culture flask. Incubate at $32{ }^{\circ} \mathrm{C}$ for 5 days.

10. Day 14: Check appearance of parasites under the microscope (400X). They should be in the pathogenic amastigote stage, lacking the flagellum characteristic of promastigotes, and ready for assay.

3. Cytotoxicity assay on L. mexicana axenic amastigotes.

NOTE: The biological testing of peptoids uses high-throughput assays carried out in 96 well plates. This protocol describes the testing of L. mexicana axenic amastigotes, but identical assays can also be carried out on the promastigote stage parasites in appropriate media. Compounds are incubated with parasites at concentrations from $3-100 \mu \mathrm{M}$ for 60 min and then incubated for $24 \mathrm{hr}$ following a 10 -fold dilution. Results are gained by measuring the fluorescence of wells after incubation with Resazurin-based cell viability solution (e.g., alamarBlue).

1. Prepare compound stock solutions. Weigh out $1 \mathrm{mg}$ of the final purified peptoid product using an analytical balance. Add the appropriate volume of molecular biology grade dimethylsulphoxide (DMSO) to a concentration of $5 \mathrm{mM}$. Make $6 \mu \mathrm{l}$ aliquots and freeze at $-20^{\circ} \mathrm{C}$

2. Prepare compound solutions on 96 well plates (a recommended plate layout can be seen in the results section, Figure 6) in triplicate from 100 to $3 \mu \mathrm{M}$. Add $2 \mu \mathrm{l}$ of $5 \mathrm{mM}$ stock solution of each compound in the top row (i.e., A). Add $48 \mu$ l fresh Schneider's Insect medium (at pH 5.5 and $20 \%$ fetal bovine serum (FBS), $1 \%$ penicillin/streptomycin(P/S)) to top row using a multi-channel pipette. Add $25 \mu \mathrm{l}$ Schneider's Insect medium to all other rows (B - F).

1. Carry out a serial dilution by pipetting $25 \mu \mathrm{l}$ solution from the top row. Add to the row below and mix. Carry out dilutions until the last row, where the last $25 \mu$ l solution should be discarded.

2. Use amphotericin $B$ ( $5 \mathrm{mM}$ stocks) as a positive control and DMSO ( $2 \%$ solution) as a negative control in triplicate.

3. Prepare parasite solution: transfer culture to a $50 \mathrm{ml}$ centrifuge tube and centrifuge at $447 \mathrm{xg}$ for $5 \mathrm{~min}$. Pour off old medium and add $10 \mathrm{ml}$ Schneider's Insect medium (at pH 5.5 and $20 \%$ FBS, $1 \% \mathrm{P} / \mathrm{S}$ ).

4. Gently dissolve the pellet of parasites in the new medium using a pipette and count using a Neubauer improved hemocytometer. Dilute culture to $8 \times 10^{6}$ parasites $/ \mathrm{ml}$.

5. Add $25 \mu \mathrm{L}$ L. mexicana culture to each well. Incubate plates for $60 \mathrm{~min}$ at $32^{\circ} \mathrm{C}$

6. Remove plate from the incubator and remove $40 \mu \mathrm{l}$ solution from each well.

7. Add $90 \mu \mathrm{l}$ fresh medium and incubate for $24 \mathrm{hr}$ at $32{ }^{\circ} \mathrm{C}$.

8. Add $10 \mu \mathrm{l}$ Resazurin-based cell viability solution to each well. Incubate for $4 \mathrm{hr}$ at $32{ }^{\circ} \mathrm{C}$

9. Measure the fluorescence using a plate reader $\left(\lambda_{\mathrm{ex}}=540 \mathrm{~nm}, \lambda_{\mathrm{em}}=600 \mathrm{~nm}\right)$, as per manufacturer's instructions. Analyze data by removing average background (from medium only wells) and comparing the fluorescence of wells after normalization with respect to the DMSO controls.

\section{Representative Results}

As a representative result, the synthesis and characterization of two 12 residue peptoids containing two lysine-type monomers and two-arginine type monomers each will be described. The subsequent results from cytotoxicity assay are also shown.

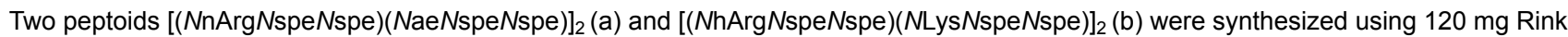
Amide resin each (loading $=0.79 \mathrm{mmol} / \mathrm{g}$ ). All acetylation and displacement steps were carried out as described above, with all reagents purchased commercially. For these residues, the following amines were used in the displacement step: Nspe (S)-(-)- $\alpha-$-methylbenzylamine, Nae $N$-(tert-butoxycarbonyl)-1,2-diaminoethane, $N$ Lys $N$-(tert-butoxycarbonyl)-1,4-diaminobutane. For the arginine derivative residues, the following unprotected diamines were coupled: NhArg 1,4-diaminobutane or NnArg 1,2-diaminoethane, followed by on-resin protection with 2-acetyldimedone (Dde-OH). After the entire sequence had been synthesized, hydrazine deprotection of the Dde yields free amines to guanidinylate. 
jove

Journal of Visualized Experiments

www.jove.com

(a)

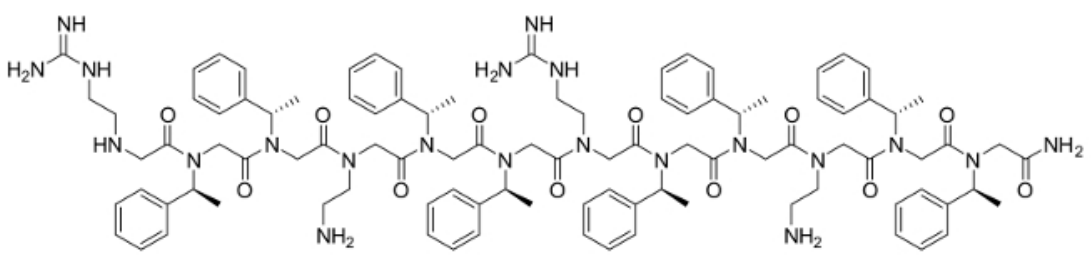

(b)

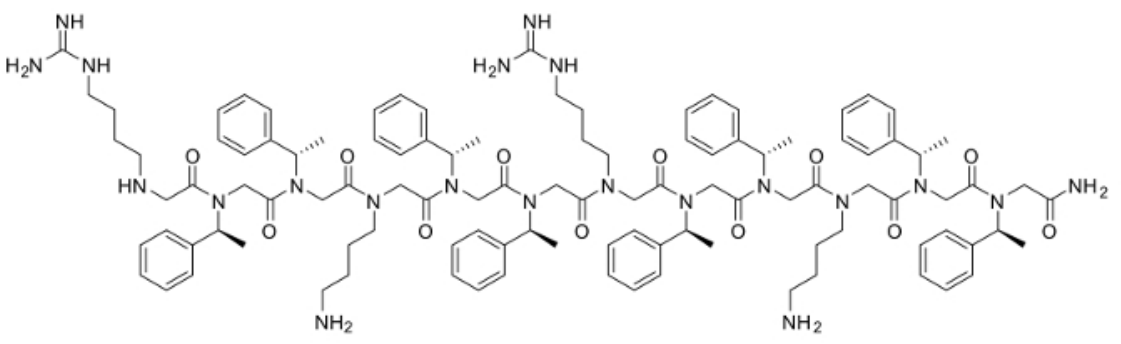

Figure 1. Peptoid structures (a) $[(N n A r g N s p e N s p e)(N a e N s p e N s p e)]_{2}$ and (b) $[(N h A r g N s p e N s p e)(N L y s N s p e N s p e)]_{2}$. Please click here to view a larger version of this figure.

Copyright @ 2016 Creative Commons Attribution 3.0 License

November 2016 | 117 | e54750 | Page 5 of 12 


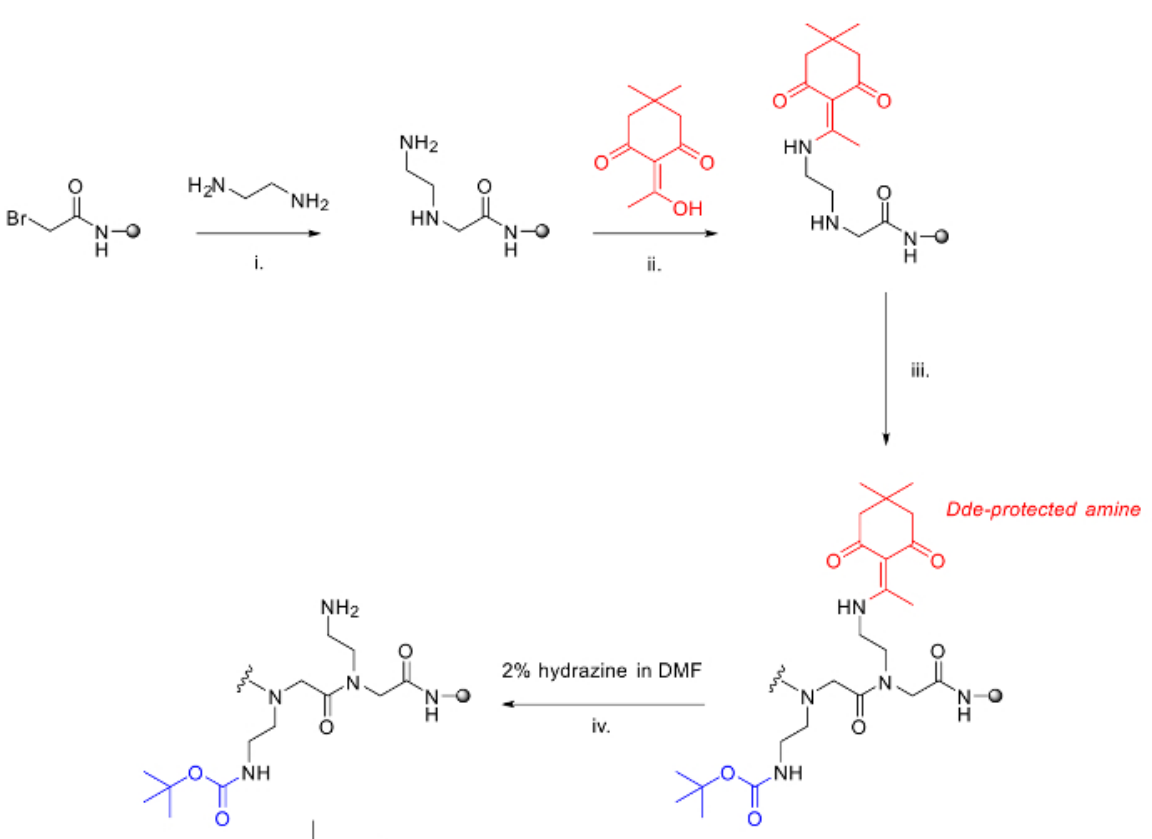<smiles>N=C(N)n1cccn1</smiles>

Boc-protected amine

\section{NArg type monomer}<smiles>CN(CCNC(=O)OC(C)(C)C)CC(=O)N(CCNC(=O)NO)CC(N)=O</smiles><smiles>NCCN(CCN)CC(=O)N(CCN)CC(N)=O</smiles>

NLys type monomer

Figure 2. The method used to synthesize mixed arginine/lysine peptoids. i. Standard displacement step in the submonomer method with diamine; ii. Addition of Dde-OH, 90 minutes to protect free amine; iii. Further additions to extend the peptoid chain using the submonomer method; iv. Deprotection of Dde using 2\% hydrazine in DMF; v. Guanidinylation of free amine on resin with pyrazole-1-carboxamidine and DIPEA in DMF; vi. Acidic cleavage from the resin and deprotection of Boc groups. Please click here to view a larger version of this figure.

Following cleavage from the resin and lyophilization, the crude products were obtained as white powders: (a) $154 \mathrm{mg}$, (b) $163 \mathrm{mg}$. Products were purified via RP-HPLC as described with maximum 50 mg injections using a LC pump with a UV-vis detector $(\lambda=250 \mathrm{~nm})$ on an analytical column, $250 \mathrm{~mm} \times 10 \mathrm{~mm}, 5 \mu \mathrm{m}$; flow rate $=2 \mathrm{ml} / \mathrm{min}$. Fractions corresponding to the target mass were combined and obtained as white powders: (a) $54 \mathrm{mg} \mathrm{(b)} 65 \mathrm{mg}$, final yields of approximately $30 \%$ for fractions $>90 \%$ pure.

The final compound identities after purification were confirmed by LC-MS (see Figure 3) using a triple quadrupole mass spectrometer equipped with an UPLC and a photodiode array detector. Accurate mass spectrometry was undertaken using the same spectrometer on the $[\mathrm{M}+2 \mathrm{H}]^{2+}$ ions. The following calculated and observed masses were found in close agreement (Figure 4): (a) calculated $=896.0026$ amu, observed $=896.0038$ amu; (b) calculated $=952.0691 \mathrm{amu}$, observed $=952.0730 \mathrm{amu}$. 
a)
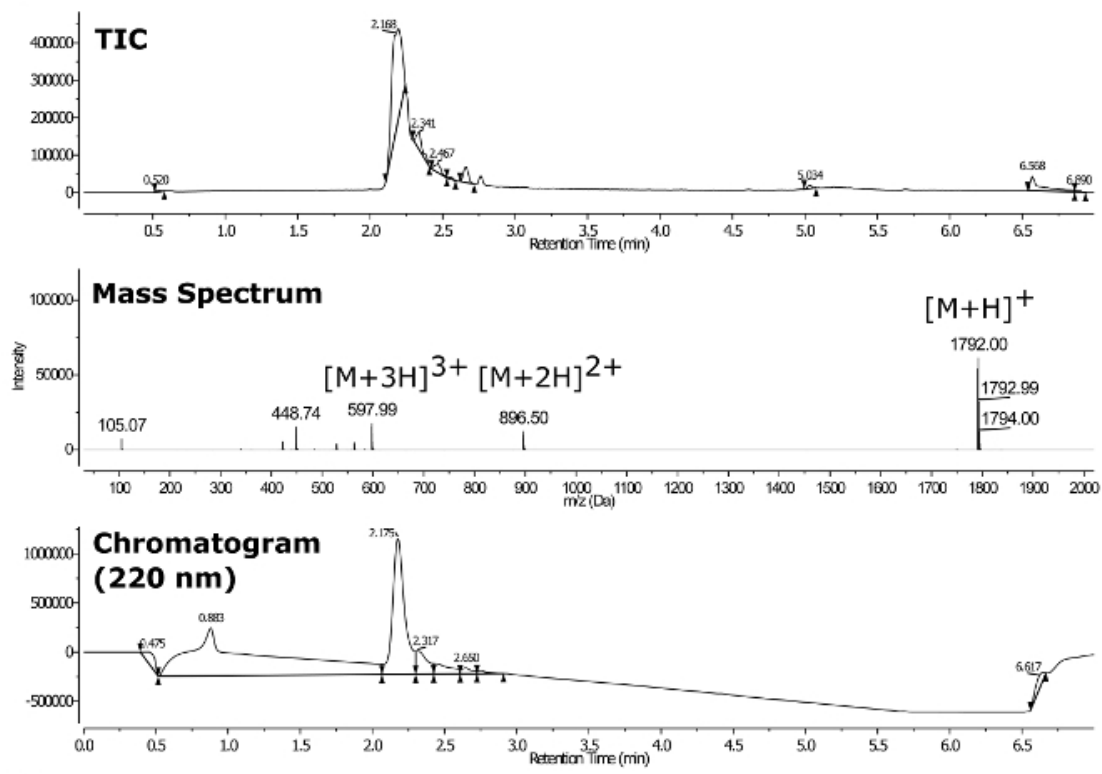

b)
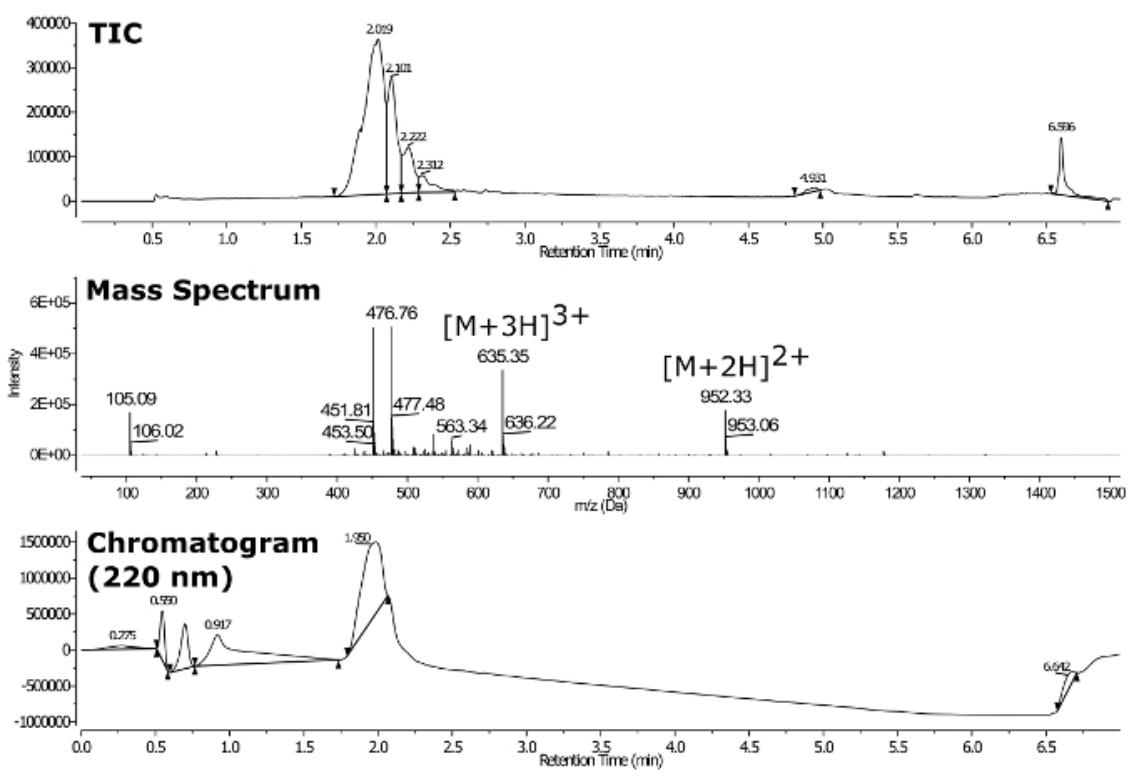

Figure 3. LC-MS for purified peptoids. (a) $\mathrm{m} / \mathrm{z}=1,792$ and (b) $\mathrm{m} / \mathrm{z}=1,903$. Where the top is TIC, middle LC-MS spectrum, bottom UV chromatogram at $220 \mathrm{~nm}$. Please click here to view a larger version of this figure. 
(a)

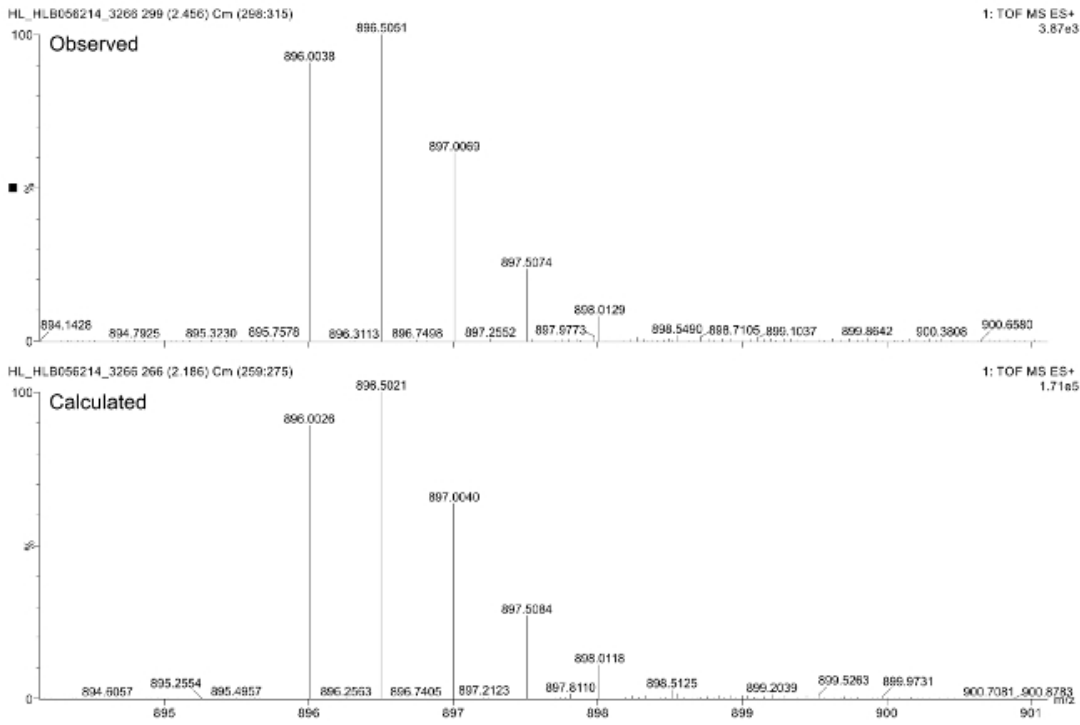

(b) HLBO2-47-11 accurate 249 (2.050) Cm (249:253)

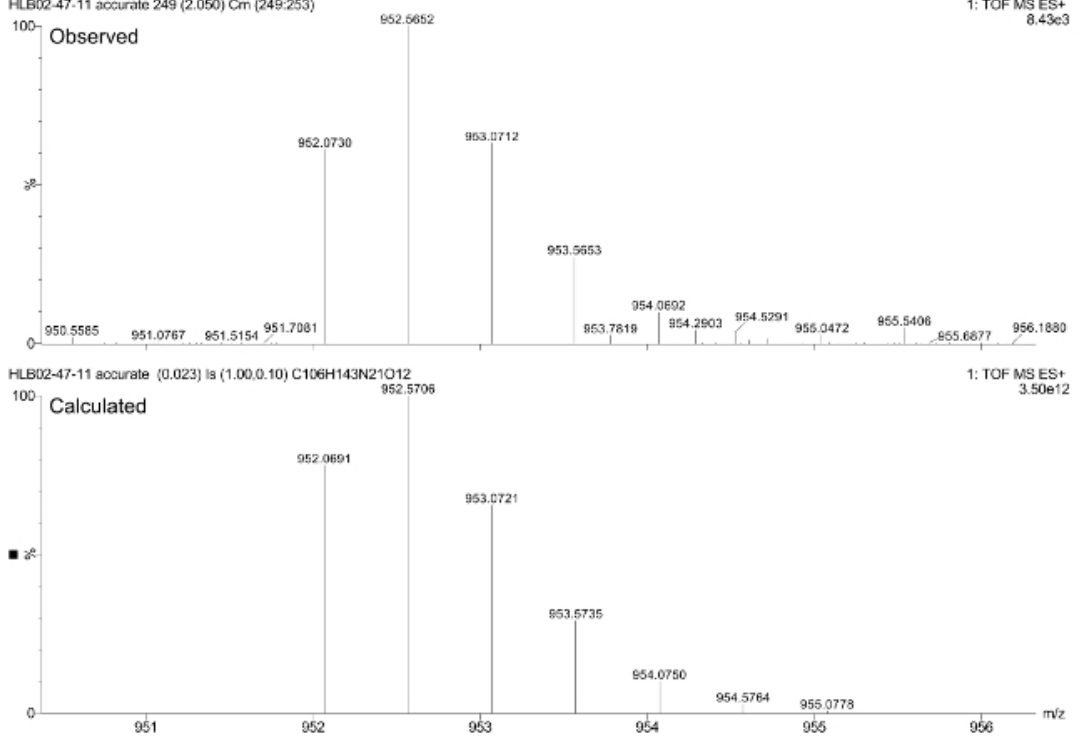

Figure 4. Accurate mass spectrometry data for peptoids (a) and (b). Please click here to view a larger version of this figure.

Product purity was assessed using an analytical RP-HPLC (LC pump with a UV-vis detector on an analytical column, $4.6 \mathrm{~mm} \times 100 \mathrm{~mm}, 3.5$ $\mu \mathrm{m}$; flow rate $=1 \mathrm{ml} / \mathrm{min}$ ), and visualized at $220 \mathrm{~nm}$, the absorbance of amide backbone. Figure $\mathbf{5 a}$ and $\mathbf{5 b}$ shows that the compounds are homogeneous. 
(a)

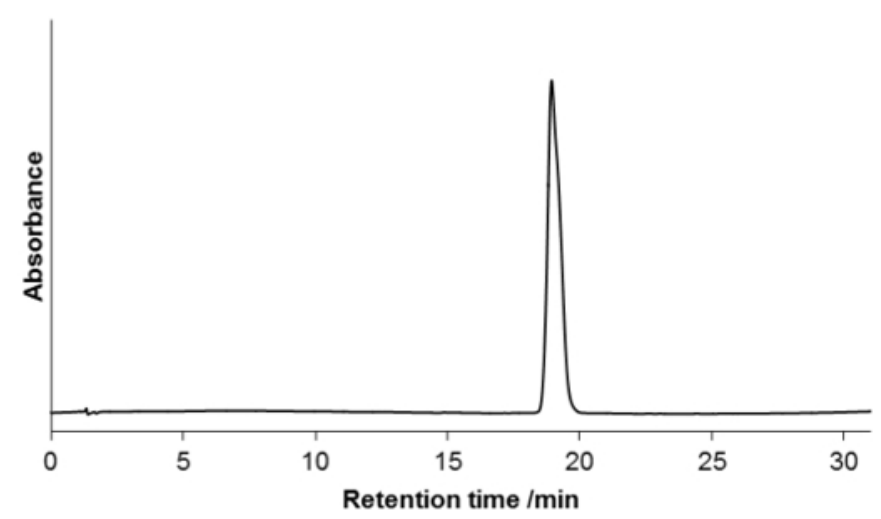

(b)

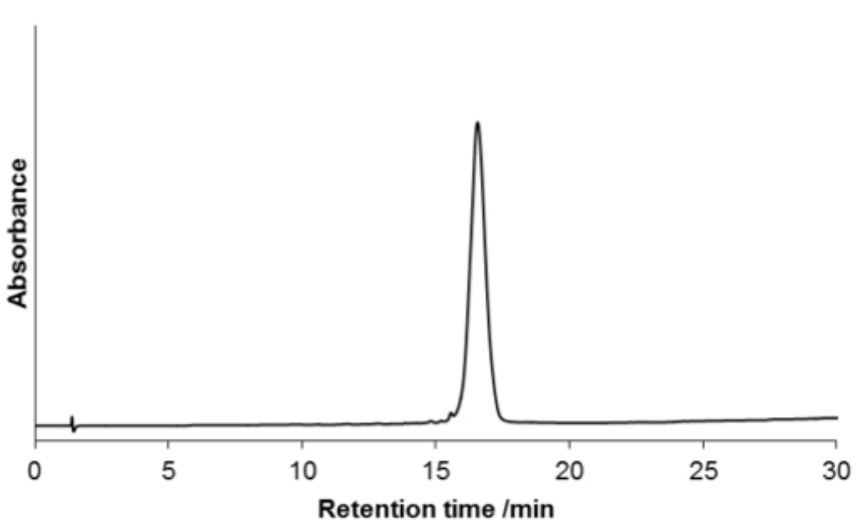

Figure 5. Analytical HPLC for the purified peptoids (a) and (b). There is a gradient $0-100 \% \mathrm{~B}$ over 30 minutes, column oven at $40{ }^{\circ} \mathrm{C}(\mathrm{A}=$ $95 \% \mathrm{H}_{2} \mathrm{O}, 5 \% \mathrm{MeCN}, 0.05 \%$ TFA; $\left.\mathrm{B}=95 \% \mathrm{MeCN}, 5 \% \mathrm{H}_{2} \mathrm{O}, 0.03 \% \mathrm{TFA}\right)$. Please click here to view a larger version of this figure.

The purified peptoids (a) and (b) were tested in cytotoxicity assays against $L$. mexicana axenic amastigotes. Frozen stocks of $L$. mexicana were defrosted and transformed to the amastigote stage ready for the assay. 72 hours after defrosting, the parasites should be insect stage promastigotes in their procyclic form, in log phase with many dividing cells. At this stage the parasites can be transformed into the amastigote stage using the $\mathrm{pH}$ and temperature shift described. ${ }^{27}$ At Day 9 of the transformation, the parasites will be in the non-replicating infectious metacyclic promastigote stage. Finally at Day 14, the parasites should be in the pathogenic amastigote stage where parasites lack the characteristic flagella of the promastigotes. ${ }^{28}$

$5 \mathrm{mM}$ stock solutions of the compounds were made in cell culture grade DMSO and tested in triplicates on a minimum of two occasions to ensure a robust data set was collected. A representative 96-well plate plan is shown in Figure 6 . At the end of the assay, the cell viability reagent was added to each well and the fluorescence was measured as described to calculate the viability of parasites at each concentration tested (see Figure 7). $E D_{50}$ values were calculated as peptoid (a) $>100 \mu \mathrm{M}$ and peptoid (b) $37 \mu \mathrm{M}$ respectively. The error bars plotted, show the variation between wells as a standard deviation and it can be seen that these are reasonable for most bars. 


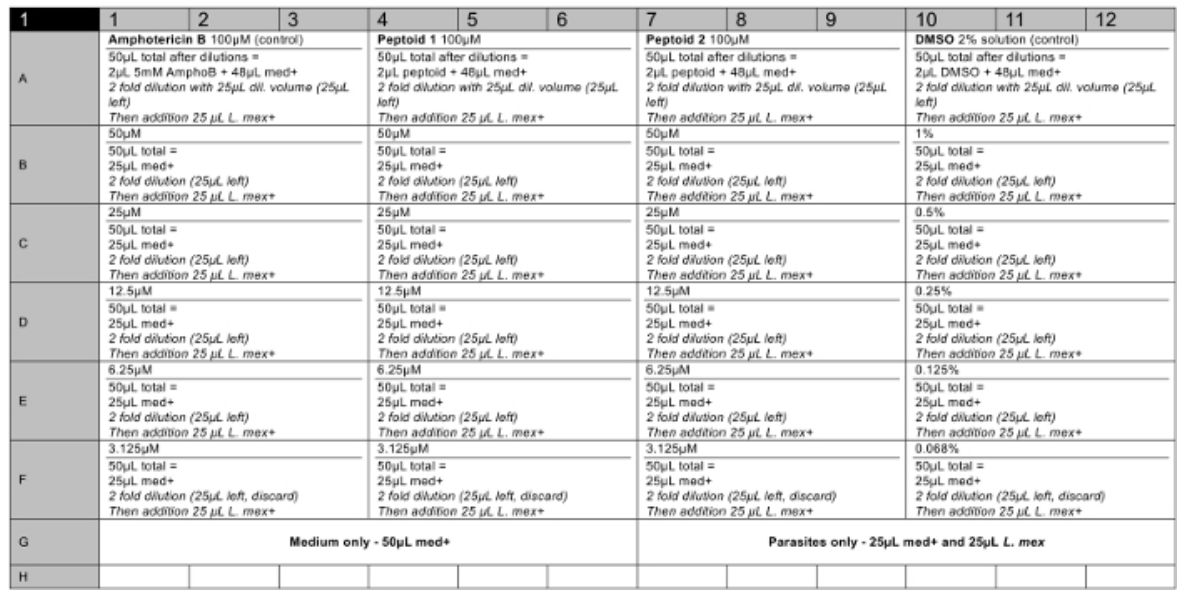

Figure 6. Representative 96 well plate plan for a cytotoxicity assay on 2 peptoid solutions (including positive and negative controls). med $+=$ medium (Schneider's Insect Medium, pH 5.5, 20\% FBS, $1 \%$ P/S). L. mex. $=8 \times 10^{6} / \mathrm{ml}$ parasite culture in med + . AmphoB $=5 \mathrm{mM}$ in DMSO. Peptoid $=5 \mathrm{mM}$ stock solution in DMSO. Empty wells should contain sterile water. Please click here to view a larger version of this figure.

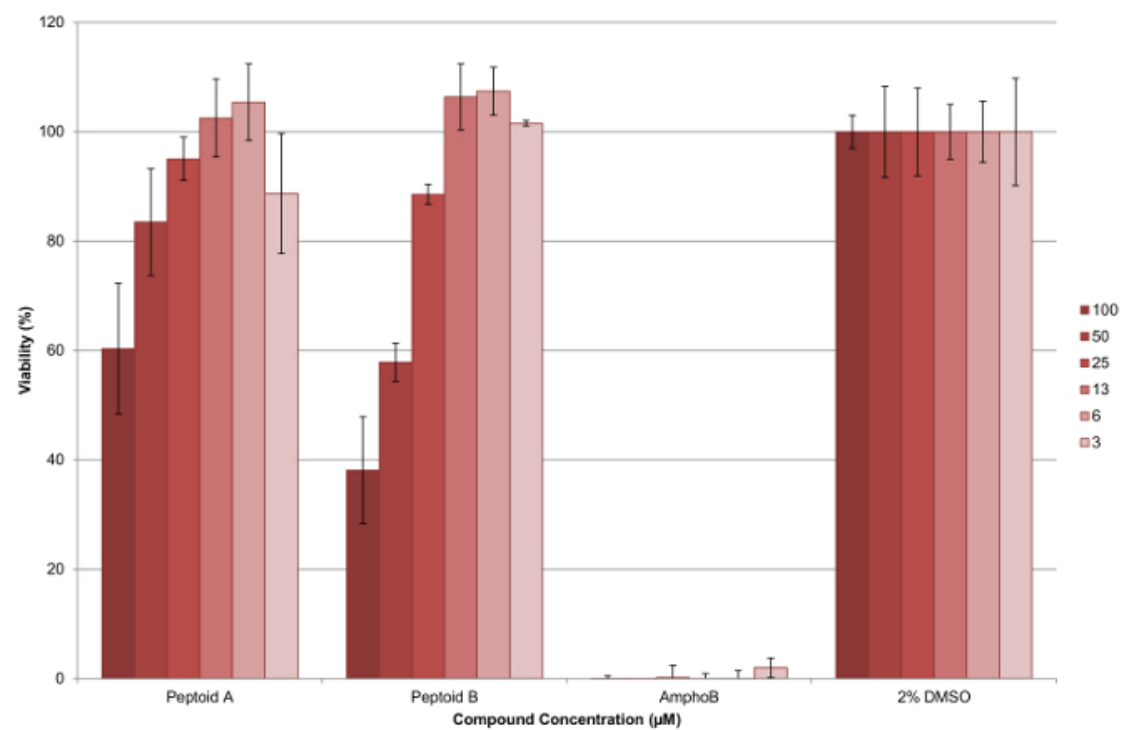

Figure 7. Results from cytotoxicity assay against $L$. mexicana axenic amastigote parasites using peptoid (a) and (b). It can be seen that the peptoid (b) is more effective than peptoid (a) at reducing the percentage of viable parasites, with both compounds having a dose dependent effect. The error bars plotted show the variation between wells as a standard deviation. Please click here to view a larger version of this figure.

\section{Discussion}

Peptoids are increasingly being studied within the chemical biology and medicinal chemistry fields in applications such as novel therapeutics ${ }^{3-5}$ cell delivery agents ${ }^{18,20}$ and diagnostic tools. ${ }^{29}$ Typically, these sequences are cationic to provide a degree of selectivity for the pathogen over mammalian cells, the ability to penetrate through cell membranes and also aid solubility in aqueous systems. There are numerous examples of cationic peptoids that contain solely lysine- or arginine-mimetic residues in the literature. However, to date, the synthesis of peptoids that contain both of these cationic residues in the same sequence has been hindered by lack of a suitable synthetic procedure. The protocol described here allows mixed cationic peptoids to be synthesized in an efficient manner and is highly desirable as it offers a route to modulate the biological and chemical properties of amphipathic peptoids.

Our method uses an adaptation to the commonly used submonomer peptoid synthesis and permits the addition of both lysine- and argininetype monomers within the same sequence. It uses room temperature couplings and established protecting group chemistry so it is anticipated that this method will be useful for the majority of research groups. To add orthogonal protection for the arginine-type residue, an unprotected diamine is added under standard displacement conditions and then protected in a 60-minute coupling with Dde-OH. A variety of diamines can be used which allows side chains from 2 carbons to 6 carbons long to be installed, i.e., 1,2-diaminoethane to 1,6-diaminohexane respectively. The protecting Dde-OH dissolves well in DMF and is a very efficient and selective protecting group for primary amines. The Dde-protection group leaves secondary amines unaffected, e.g., the unprotected $\mathrm{N}$ terminus of peptoid chain. ${ }^{30}$ One limitation is that all of the synthesis was 
undertaken manually; however, it is anticipated that the coupling conditions developed make the method amenable for use with automated peptide/peptoid synthesizers.

The on resin deprotection of the Dde-groups is undertaken using a $2 \%$ hydrazine solution in DMF to leave free amines that can be guanidinylated on resin using pyrazole-1-carboxamidine. Six equivalents of the pyrazole-1-carboxamidine and six equivalents of DIPEA are used per free amine on the resin (i.e., six equivalents for each NArg type monomer to be installed). Again, this reaction is also efficient and the pyrazole-1-carboxamidine reagent has good solubility in DMF. Completion of the reaction is typically seen via LC-MS after 60 minutes at room temperature.

Due to the versatility of the submonomer method, a wide variety of primary amines can be used in the displacement step so conditions may need to be optimized to increase coupling efficiency and overall product yields or purity. ${ }^{31}$ For the sequences discussed above, no special conditions were necessary for successful couplings. However, longer displacement times or higher amine concentrations could be used for problematic displacements (i.e., for poorly nucleophilic or sterically bulky amines). Some amines may not be completely soluble in DMF, in which case it is recommended to dissolve these in N-methyl-2-pyrrolidone (NMP), or other appropriate solvents for solid-phase reactions instead as in a previous comprehensive method for submonomer synthesis of peptoids. ${ }^{32}$ To incorporate monomers that contain unprotected heteroatoms in the side chains, acetylation using chloroacetic acid has been shown to be effective by other groups. ${ }^{33}$ Additionally, other resins may be used with this method to yield peptoids with different $C$ terminal functionalization. Wang resins and 2-chlorotrityl chloride resins are routinely used in the submonomer synthesis of peptoids. For example, this method has been successfully used with 2-chlorotrityl chloride resin in our group. ${ }^{2}$ Different solid supports will require a different loading procedure to Rink Amide discussed here (dependent on the specific resin used) so this should be checked with the literature prior to synthesis.

Similar to peptide synthesis, the conditions for final cleavage of the peptoid off resin can also be optimized for the specific sequence. In this protocol, a TFA cleavage cocktail was used (with triisopropylsilane and water as scavengers). The peptoids presented here contained only Boc protection which is a reasonably acid labile group. To ensure complete deprotection of sequences with a greater proportion of protected residues or less acid labile protecting groups, longer cleavage times may be necessary (i.e., cleavage times in excess of 2 hours are recommended for sequences that contain Pbf or tertiary-butyl ester protected groups). Alternative scavengers can also be used for specialized side chains (for example, ethanedithiol or 2-mercaptoethanol are often used in peptides that have sulphur containing side chains like cysteine or methionine).

The biological assay presented is a standard cytotoxicity test which can be altered to suit different cell lines. It is important to note that each 96well plate should contain sufficient controls to allow confidence in the results obtained. In this case, amphotericin B is used as a positive control as it is a known drug used to treat the disease and DMSO is used as a negative control as this is the solvent used to make compound stocks for the assay. If other cell lines are being used, alternative, suitable controls should be obtained and validated before use. $L$. mexicana is incubated with the peptoid at concentrations between 100 and $2 \mu \mathrm{M}$ for one hour, and then the parasite/peptoid solution is diluted by a factor of ten for overnight incubation (i.e., wells initially with $100 \mu \mathrm{M}$ peptoid stock are diluted to $10 \mu \mathrm{M}$ ).

The cell viability reagent is added to each well ( $10 \%$ of total well volume) at the end of the assay. A visible color change is seen between wells with viable parasites (pink), control wells with no viable parasites (blue) and a spectrum between with intermediate numbers of viable parasites. The fluorescence is proportional to the number of living cells and corresponds to the metabolic activity of the cells; resazurin dye (nonfluorescent) is converted to the fluorescent resorufin by reduction reactions in metabolically active cells. ${ }^{34}$ In this assay, the incubation time with the cell viability reagent has been optimized for $L$. mexicana. Incubation times with the viability reagent will vary for plates seeded at different cell concentrations or with different cell lines (for example this method can also be used with mammalian cells). Dependent on the exact plate reader used, considerations need to be made before fluorescence measurements are taken. Any air bubbles in wells should be removed to ensure accurate readings can be taken. Some plate readers read from the bottom of plates, in which case flat bottomed 96 well plates should be used. Other machines may read from the top of the plate, so the lid of the plate should be removed before measurement.

Finally, in the future, this protocol may be compatible with automated synthesizers that are capable of making many sequences in parallel. Additionally, the synthesis of cyclic peptoids is also possible using this method. This protocol should provide researchers with a practical synthetic procedure that can be used to access novel peptoid scaffolds with both lysine- and arginine-type monomers, which may be of use in many applications, including materials or medicinal fields.

\section{Disclosures}

The authors have nothing to disclose.

\section{Acknowledgements}

We thank the Engineering and Physical Sciences Research Council (EPSRC) for financial support (HLB). We also thank Sridévi Maalika Ramanoudjame for her assistance during the filming of this procedure.

\section{References}

1. Zuckermann, R. N., Kerr, J. M., Kent, S. B. H., \& Moos, W. H. Efficient method for the preparation of peptoids oligo(N-substituted glycines) by submonomer solid-phase synthesis. J. Am. Chem. Soc. 114, 10646-10647 (1992).

2. Bolt, H. L., \& Cobb, S. L. A practical method for the synthesis of peptoids containing both lysine-type and arginine-type monomers. Org. Biomol. Chem. 14, 1211-1215 (2016).

3. Mojsoska, B., Zuckermann, R. N., \& Jenssen, H. Structure-activity relationship study of novel peptoids that mimic the structure of antimicrobial peptides. Antimicrob Agents Chemother. (2015). 
4. Chongsiriwatana, N. P. et al. Peptoids that mimic the structure, function, and mechanism of helical antimicrobial peptides. Proc. Natl. Acad. Sci. USA. 105, 2794-2799 (2008).

5. Eggimann, G. A., Bolt, H. L., Denny, P. W., \& Cobb, S. L. Investigating the Anti-leishmanial Effects of Linear Peptoids. Chem Med Chem. 10, 233-237 (2015).

6. Kapoor, R. et al. Antimicrobial Peptoids Are Effective against Pseudomonas aeruginosa Biofilms. Antimicrob. Agents Chemother. 55, 3054-3057 (2011).

7. Ryge, T. S., Frimodt-Moller, N., \& Hansen, P. R. Antimicrobial activities of twenty lysine-peptoid hybrids against clinically relevant bacteria and fungi. Chemotherapy. 54, 152-156 (2008).

8. Huang, M. L., Benson, M. A., Shin, S. B. Y., Torres, V. J., \& Kirshenbaum, K. Amphiphilic Cyclic Peptoids That Exhibit Antimicrobial Activity by Disrupting Staphylococcus aureus Membranes. Eur. J. Org. Chem. 3560-3566 (2013).

9. Huang, M. L., Shin, S. B. Y., Benson, M. A., Torres, V. J., \& Kirshenbaum, K. A Comparison of Linear and Cyclic Peptoid Oligomers as Potent Antimicrobial Agents. Chem Med Chem. 7, 114-122 (2012).

10. Bolt, H. L., Eggimann, G. A., Denny, P. W., \& Cobb, S. L. Enlarging the Chemical Space of Anti-leishmanials: a Structure-Activity Relationship Study of Peptoids against Leishmania mexicana, a Causative Agent of Cutaneous Leishmaniasis. Med Chem Comm. (2016).

11. The World Health Organisation. Leishmaniasis. <http://www.who.int/leishmaniasis/en/> (2016).

12. Chon, S. Y. et al. Antibiotic overuse and resistance in dermatology. Dermatologic therapy. 25, 55-69 (2012).

13. Alvar, J. et al. Leishmaniasis Worldwide and Global Estimates of Its Incidence. PLOS ONE. 7 (2012).

14. Croft, S. L., Barrett, M. P., \& Urbina, J. A. Chemotherapy of trypanosomiases and leishmaniasis. Trends Parasitol. 21, 508-512 (2005).

15. Kedzierski, L. Leishmaniasis Vaccine: Where are We Today? J Global Infect Dis. 2, 177-185 (2010).

16. Croft, S. L., Sundar, S., \& Fairlamb, A. H. Drug resistance in leishmaniasis. Clin Microbiol Rev. 19, 111-126 (2006).

17. Huang, W. et al. Learning from Host-Defense Peptides: Cationic, Amphipathic Peptoids with Potent Anticancer Activity. PLOS ONE. 9 (2014).

18. Wender, P. A. et al. The Design, Synthesis, and Evaluation of Molecules That Enable or Enhance Cellular Uptake: Peptoid Molecular Transporters. Proc. Natl. Acad. Sci. USA. 97, 13003-13008 (2000).

19. Schröder, T. et al. Peptoidic Amino- and Guanidinium-Carrier Systems: Targeted Drug Delivery into the Cell Cytosol or the Nucleus. J. Med. Chem. 51, 376-379 (2008).

20. Kömel, D. K. et al. Cell-penetrating peptoids: Introduction of novel cationic side chains. Eur. J. Med. Chem. 79, 231-243 (2014).

21. Hein-Kristensen, L., Knapp, K. M., Franzyk, H., \& Gram, L. Bacterial membrane activity of $\alpha$-peptide/ $\beta$-peptoid chimeras: Influence of amino acid composition and chain length on the activity against different bacterial strains. BMC Microbiology. 11, 1-12 (2011).

22. Su, Y., Doherty, T., Waring, A. J., Ruchala, P., \& Hong, M. Roles of Arginine and Lysine Residues in the Translocation of a Cell-Penetrating Peptide from (13)C, (31)P and (19)F Solid-State NMR. Biochem. 48, 4587-4595 (2009).

23. Andreev, K. et al. Guanidino groups greatly enhance the action of antimicrobial peptidomimetics against bacterial cytoplasmic membranes. BBA Biomembranes. 1838, 2492-2502 (2014).

24. Foged, C. et al. Cellular uptake and membrane-destabilising properties of alpha-peptide/beta-peptoid chimeras: lessons for the design of new cell-penetrating peptides. BBA Biomembranes. 1778, 2487-2495 (2008).

25. Vedel, L. et al. Antiplasmodial and prehemolytic activities of alpha-peptide-beta-peptoid chimeras. Chem Bio Chem. 8, 1781-1784 (2007).

26. Lear, S., \& Cobb, S. L. Pep-Calc.com: a set of web utilities for the calculation of peptide and peptoid properties and automatic mass spectral peak assignment. J Computer-Aided Mol Des., 30, 271 (2016).

27. Bates, P. A. Complete developmental cycle of Leishmania mexicana in axenic culture. Parasitol. 108, 1 - 9 (1994)

28. Gossage, S. M., Rogers, M. E., \& Bates, P. A. Two separate growth phases during the development of Leishmania in sand flies: implications for understanding the life cycle. Int J Parasitology. 33, 1027-1034 (2003).

29. Seo, J., Lee B.C., \& Zuckermann, R.N. in Comp Biomaterials. Vol. 2 (eds P. Ducheyne et al.) 53-76, Elsevier (2011).

30. Nash, I. A., Bycroft, B. W., \& Chan, W. C. Dde - A selective primary amine protecting group: A facile solid phase synthetic approach to polyamine conjugates. Tetrahedron Lett. 37, 2625-2628 (1996).

31. Culf, A. S., \& Ouellette, R. J. Solid-phase synthesis of N-substituted glycine oligomers (alpha-peptoids) and derivatives. Molecules. 15, 5282-5335 (2010).

32. Tran, H., Gael, S. L., Connolly, M. D., \& Zuckermann, R. N. Solid-phase Submonomer Synthesis of Peptoid Polymers and their Self-Assembly into Highly-Ordered Nanosheets, J Vis Exp. e3373 (2011).

33. Burkoth, T. S., Fafarman, A. T., Charych, D. H., Connolly, M. D., \& Zuckermann, R. N. Incorporation of unprotected heterocyclic side chains into peptoid oligomers via solid-phase submonomer synthesis. J. Am. Chem. Soc. 125, 8841-8845 (2003).

34. Nakayama, G. R., Caton, M. C., Nova, M. P., \& Parandoosh, Z. Assessment of the Alamar Blue assay for cellular growth and viability in vitro. J Immunol Methods. 204, 205-208 (1997). 